\title{
ASYMPTOTIC PERFORMANCE OF A MULTISTATE COHERENT SYSTEM
}

\author{
SRINIVAS IYER, * Indian Statistical Institute, New Delhi
}

\begin{abstract}
An expression for the asymptotic or steady-state performance function is derived for a multistate coherent system when each component changes states in time according to a semi-Markov process, the stochastic processes being mutually independent. This generalizes the expression for system availability of a binary coherent system when the components are governed by mutually independent alternating renewal processes.
\end{abstract}

SEMI-MARKOV PROCESS; ASYMPTOTIC PERFORMANCE

\section{Introduction}

El-Neweihi et al. [3] consider a multistate coherent system which is a natural generalization of a binary coherent system. Here a dynamic version of the system is considered and an expression for the asymptotic performance function is derived when each component changes states in time according to a semi-Markov process, the stochastic processes being mutually independent. The result generalizes that for system availability in [1] where the states of the components are governed by mutually independent alternating renewal processes.

In Section 2 the notation and description of a multistate coherent system is given, along with a definition of the performance function of the system. In Section 3 the dynamic semi-Markov model is defined and an expression for the asymptotic performance function derived, while in Section 4 the steady-state expression is derived for a special case of a multistate coherent system due to Barlow and $\mathrm{Wu}[2]$.

\section{Notation and description of a multistate coherent system}

The notation and description of the system is as in [3]. For each component and for the system itself we can distinguish among $M+1$ states representing successive levels of performance ranging from perfect functioning (level $M$ ) down to complete failure (level 0 ). For component $i, x_{i}$ denotes the corresponding state or performance level, $i=1, \ldots, n$; the vector $\boldsymbol{x}=\left(x_{1}, \ldots, x_{n}\right)$ denotes the vector of states of components $1, \ldots, n$. We assume that the state $\Phi$ of the system is a deterministic function of the states $x_{1}, \ldots, x_{n}$ of the components. Thus $\Phi=\phi(x)$, where $\boldsymbol{x}$ takes values in $S^{n}, S=\{0,1, \ldots, M\}$ and $\Phi$ takes value in $S$.

The multistate coherent system (MCS) considered in [3] is a natural generalization of a binary coherent system and is defined there as follows. Let

$$
\begin{aligned}
& \left(j_{i}, \boldsymbol{x}\right)=\left(x_{1}, \ldots, x_{i-1}, j, x_{i+1}, \ldots, x_{n}\right) \text { where } j=0,1, \ldots, M \\
& (., \boldsymbol{x})=\left(x_{1}, \ldots, x_{i-1}, \ldots, x_{i+1}, \ldots, x_{n}\right) \text { and } j=(j, j, \ldots, j) .
\end{aligned}
$$

Received 21 May 1987; revision received 28 September 1987.

* Postal address: Indian Statistical Institute, Statistical Quality Control and Operations Research Unit, 7 S.J.S. Sansanwal Marg, New Delhi 110016, India. 
A system of components is said to be an MCS if its structure function $\Phi$ satisfies:

(i) $\Phi$ is increasing (in each argument),

(ii) For level $j$ of component $i$, there exists a vector $\left({ }_{i}, \boldsymbol{x}\right)$ such that $\Phi\left(j_{i}, \boldsymbol{x}\right)=j$ while $\Phi\left(l_{i}, \boldsymbol{x}\right) \neq j$ for $l \neq j, i=1, \ldots, n$ and $j=0,1, \ldots, M$.

(iii) $\Phi(j)=j$ for $j=0,1, \ldots, M$.

Condition (ii) may be replaced by either of the two weaker coherency conditions mentioned in Griffith [4] without affecting any of the results to follow.

In [3] the performance function of the system is defined which is a generalization of the concept of reliability for a binary system.

Let $X_{i}$ denote the random state of component $i=1, \ldots, n$, with

$$
P\left[X_{i}=j\right]=P_{i j}, \quad P\left[X_{i} \leqq j\right]=P_{i(j)}, \quad P\left[X_{i} \geqq j\right]=Q_{i(j)},
$$

where $j=0,1, \ldots, M . P_{i}$ represents the performance distribution of component $i$. Let $\boldsymbol{X}=\left(X_{1}, \ldots, X_{n}\right)$ be the random vector representing the states of components $1, \ldots, n$ where $X_{1}, \ldots, X_{n}$ are assumed to be statistically mutually independent. Then $\Phi(X)$ is the random variable representing the system state of the MCS having structure function $\Phi$, with

$$
P[\Phi(X)=j]=P_{j}, \quad P[\Phi(X) \leqq j]=P(j), \quad j=0,1, \ldots, M .
$$

$P$ represents the performance distribution of the system.

In [3] the performance function $h$ of the system is defined as

$$
h=h_{p}\left(\boldsymbol{p}_{1}, \ldots, \boldsymbol{p}_{n}\right)=E[\Phi(\boldsymbol{x})]
$$

where $\boldsymbol{p}_{i}=\left(p_{i 0}, \ldots, p_{i M}\right)$ and $\boldsymbol{p}=\left(\boldsymbol{p}_{1}, \ldots, \boldsymbol{p}_{n}\right), i=1, \ldots, n$.

\section{A dynamic semi-Markov model}

We consider a dynamic version of the system and study the asymptotic performance function $h$.

Each component changes states in time according to a semi-Markov process (SMP), the stochastic processes being mutually independent. The SMP for component $i$ has parameters $\left\{\Pi_{j}^{i}, \mu_{j}^{i}, \mu_{j j}^{i}, j=0,1, \ldots, M\right\}$ (see [6]), where $\Pi_{j}^{i}$ is the steady-state probability of state $j$ for the embedded Markov chain of SMP ${ }^{i}, \mu_{j}^{i}$ is the mean time in state $j$ of $\mathrm{SMP}^{i}$, and $\mu_{j j}^{i}$ is the mean-cycle time for state $j$ of SMP.

Let $X_{i}^{t}$ denote the state of component $i$ at time $t$ with $p_{i j}^{t}=\operatorname{Pr}\left[X_{i}^{t}=j\right], i=1, \ldots, n$; $j=0,1, \ldots, M$. Then [6], $p_{i j}^{t} \rightarrow p_{i j}^{*}$, as $t \rightarrow \infty$, where $p_{i j}^{*}$ is the steady-state probability of being in state $j$ for component $i$ and is given by

$$
p_{i j}^{*}=\frac{\mu_{j}^{i}}{\mu_{j j}^{i}}=\frac{\Pi_{j}^{i} \mu_{j}^{i}}{\sum_{k=0}^{M} \Pi_{k}^{i} \mu_{k}^{i}} .
$$

For a continuous-time Markov chain these could be calculated from the rate or balance equations.

Then since [3], $h(\boldsymbol{p})$ is continuous (in fact, differentiable) with respect to $p$, see [3], $h\left(\boldsymbol{p}^{t}\right) \rightarrow h\left(\boldsymbol{p}^{*}\right)$, as $t \rightarrow \infty$, where $\boldsymbol{p}^{*}=\left(\boldsymbol{p}_{1}^{*}, \ldots, \boldsymbol{p}_{n}^{*}\right)$ is the vector of steady-state probabilities

$$
p_{k}^{*}=\left(p_{k 0}^{*}, \ldots, p_{k M}^{*}\right), \quad k=1, \ldots, n .
$$

Thus the asymptotic system performance function, $h^{*}(\boldsymbol{p})$ is given by

$$
h^{*}(\boldsymbol{p})=h\left(\boldsymbol{p}^{*}\right)=h\left(\frac{\mu_{0}^{1}}{\mu_{00}^{1}}, \frac{\mu_{1}^{1}}{\mu_{11}^{1}}, \ldots, \frac{\mu_{M}^{1}}{\mu_{M M}^{1}}, \ldots, \frac{\mu_{0}^{n}}{\mu_{00}^{n}}, \frac{\mu_{1}^{n}}{\mu_{11}^{n}}, \ldots, \frac{\mu_{M}^{n}}{\mu_{M M}^{n}}\right)
$$

where each $\mu_{j j}^{i}=\sum_{k} \Pi_{k}^{i} \mu_{k}^{i} / \Pi_{j}^{i}, i=1, \ldots, n, j=0,1, \ldots, M$. This is a generalization of the result mentioned in [1] for the system availability for a coherent binary system of $n$ 
components with structure function $\Phi$ and reliability $h_{\phi}$ governed by $n$ mutually independent ARPs, namely, system availability

$$
h^{\prime}=h\left(\frac{\mu_{1}}{\mu_{1+v_{1}}}, \ldots, \frac{\mu_{n}}{\mu_{n+v_{n}}}\right)
$$

where $\mu_{i}$ is the mean time in state 0 ('on state') for component $i$ and $v_{i}$ is the mean time in state 1 ('off state') for component $i, i=1, \ldots, n$.

\section{The Barlow-Wu model}

As a special case we consider the MCS studied in [2]. Here we have $p$ min-path sets $\left\{P_{1}, \ldots, P_{p}\right\}$ defined as for a coherent binary system. The system state function $\Phi(x)$ for the MCS is defined by

$$
\Phi(\boldsymbol{x})=\max _{1 \leqq r \leqq p} \min _{i \in P_{r}} x_{i} .
$$

Let $\Psi$ represent the coherent structure function (as in the binary case) corresponding to the min-path sets $\left\{P_{1}, \ldots, P_{p}\right\}$, and let $h_{\Psi}$ represent the reliability polynomial (as in the binary case) corresponding to $\Psi$. Then, as shown in [2],

$$
P[\Phi(\boldsymbol{x}) \geqq j]=\dot{h_{\Psi}}\left(\boldsymbol{Q}_{j}\right), \quad \boldsymbol{Q}_{j}=\left(Q_{1(j)}, \ldots, Q_{n(j)}\right) .
$$

Hence the performance function $h_{\phi}$, or simply $h$ for the MCS as defined in [2] is in this case given by

$$
h(\boldsymbol{p})=\sum_{j=1}^{M} P[\Phi(\boldsymbol{X}) \geqq j]=\sum_{j=1}^{M} h_{\Psi}\left(\boldsymbol{Q}_{j}\right)
$$

For the dynamic version of the model in [2], since, as $t \rightarrow \infty$,

$$
\boldsymbol{Q}_{j}^{t}=\left(\sum_{k=j}^{M} p_{1 k}^{t}, \ldots, \sum_{k=j}^{M} p_{n k}^{t}\right) \rightarrow\left(\sum_{k=j}^{M} p_{1 k}^{*}, \ldots, \sum_{k=j}^{M} p_{n k}^{*}\right)
$$

the asymptotic system performance function, $h(\boldsymbol{p})$ is in this case given by

$$
h^{*}(\boldsymbol{p})=\sum_{j=1}^{M} h_{\Psi}\left(\sum_{k=j}^{M} p_{1 k}^{*}, \ldots, \sum_{k=j}^{M} p_{n k}^{*}\right)
$$

or

$$
\sum_{j=1}^{M} h_{\Psi_{j}}\left(\sum_{k=j}^{M} p_{1 k}^{*}, \ldots, \sum_{k=j}^{M} p_{n k}^{*}\right)
$$

if $\psi$ varies with $j$ in the more general model of Natvig [5].

\section{References}

[1] Barlow, R. E. and Proschan, F. (1981) Statistical Theory of Reliability and Life Testing. To Begin With, Silver Spring, Md.

[2] Barlow, R. E. AND WU, A. S. (1978) Coherent systems with multistate components. Math. Operat. Res. 3, 275-281.

[3] El-Neweihi, E., Proschan, F. and Sethuraman, J. (1978) Multistate Coherent Systems. J. Appl. Prob. 15, 675-688.

[4] Griffith, W. S. (1980) Multistate reliability models. J. Appl. Prob. 17, 735-744.

[5] Natvig, B. (1982) Two suggestions of how to define a multistate coherent system. Adv. Appl.

Prob. 14, 434-455.

[6] Ross, S. M. (1982) Stochastic Processes. Wiley, New York. 